\title{
Fatores de sucesso do relacionamento entre agricultores e indústria de sementes de milho
}

\author{
Successful factors of the relationship between farmers and the corn seed \\ industry
}

\author{
Jaciene Arantes Lopes ${ }^{1}$ (D), José Márcio de Carvalho ${ }^{1}$, Fabrício Oliveira Leitão ${ }^{1}$ (1) \\ ${ }^{1}$ Programa de Pós-graduação em Agronegócios, Universidade de Brasília (UnB), Brasília (DF), Brasil. E-mails: \\ jacieneao@hotmail.com; jmcarvalho1708@hotmail.com; fabriciofol@hotmail.com
}

\begin{abstract}
Como citar: Lopes, J. A., Carvalho, J. M., \& Leitão, F. O. (2021). Fatores de sucesso do relacionamento entre agricultores e indústria de sementes de milho. Revista de Economia e Sociologia Rural, 59(4), e229897. https://doi.org/10.1590/1806-9479.2021.229897
\end{abstract}

Resumo: O presente estudo analisa a interação e as práticas de gestão da qualidade que caracterizam a relação entre os agricultores responsáveis pela produção de sementes de milho (fornecedores) e a indústria produtora de sementes (compradores). O objetivo do trabalho foi analisar a influência dos fatores relacionais - que incluem a confiança, a comunicação, a capacidade de adaptação, a relação de dependência e o nível de satisfação no desenvolvimento de uma parceria bem-sucedida, representada pelo comprometimento e pela orientação em longo prazo - e, consequentemente, a influência da parceria para a adoção de práticas de gestão da qualidade a partir da perspectiva dos agricultores. Foi realizada uma pesquisa aplicada e descritiva com abordagem quali-quantitativa. A coleta de dados ocorreu por meio da aplicação de 69 questionários a agricultores fornecedores de sementes. Para analisar o modelo teórico proposto, foi realizada uma análise fatorial confirmatória. As hipóteses foram testadas através da análise de trilhas. Os resultados indicam que uma parceria bem-sucedida é altamente influenciada pela satisfação e pela troca de informações (comunicação), e que a parceria contribui para a adoção de práticas de gestão da qualidade.

Palavras-chave: gestão da qualidade, relação comprador-fornecedor, sementes de milho.

\begin{abstract}
This study analyzes the interaction and quality management practices that characterize the relationship between farmers responsible for corn seed production (suppliers) and the seed industry (buyers). The influence of the following relational factors was analyzed: trust, communication, adaptability, dependency relationship, level of satisfaction in developing a successful partnership, represented by commitment and long-term orientation, and consequently the influence of the partnership to adopt quality management practices from the farmers' perspective. A qualitative and quantitative descriptive and applied research was performed. Data were collected from 69 seed suppliers through the application of questionnaires. Confirmatory factor analysis was performed to analyze the proposed theoretical model. The hypotheses were tested by trail analysis. The results indicate that a successful partnership is highly influenced by satisfaction and information exchange (communication), and that the partnership contributes to adopt quality management practices.
\end{abstract}

Keywords: quality management, buyer-supplier relationship, corn seeds.

\section{Introdução}

O agronegócio brasileiro é responsável por conceder ao Brasil o destaque mundial na produção de alimentos, especialmente por estar entre os maiores exportadores de commodities. A soja e o milho são os principais produtos responsáveis pela liderança do país, tanto em produção quanto em produtividade, respondendo por $84 \%$ da área total semeada com grãos no país (Brasil, 2018). 
O aumento na utilização de sementes melhoradas contribuiu para a expansão e diversificação da produção de sementes, resultantes da evolução do melhoramento genético, do uso da biotecnologia e da incorporação de novas tecnologias ao processo de produção, disponibilizando sementes de alta qualidade, de diferentes variedades, e para diferentes condições edafoclimáticas, que possibilitaram o aumento da produtividade no campo. Além disso, a produção de sementes é responsável por garantir a sustentabilidade de todo o sistema agroalimentar (Bonny, 2017).

Em 2016, o mercado brasileiro de sementes girava em 10 bilhões de reais, estando atrás somente dos Estados Unidos e da China (Associação Brasileira de Sementes e Mudas, 2016). Assim como qualquer outro segmento, apesar de suas particularidades, a produção de sementes requer a cooperação de seus membros. O desenvolvimento de parcerias entre compradores e fornecedores já é reconhecido como primordial para a competitividade das empresas (Yu \& Huo, 2018).

Neste contexto, para além dos fatores de relacionamento, uma atitude colaborativa, voltada para o gerenciamento da qualidade, também emerge como fator determinante dos relacionamentos bem-sucedidos, pois os produtos gerados pela agroindústria dependem substancialmente da eficácia dos relacionamentos com fornecedores, de modo que as empresas que buscam a melhoria da qualidade necessitam construir relacionamentos mutuamente benéficos (Dudin et al., 2015).

Para que ocorra a produção de sementes, as agroindústrias necessitam de agricultores que tenham disponibilidade de terras e outros recursos (irrigação, máquinas, conhecimento, entre outros) para que multipliquem as sementes em suas propriedades, caracterizando-se, portanto, como uma relação de troca entre fornecedores (agricultores) e compradores (indústria produtora de sementes), a qual geralmente se dá mediante contratos. Os compradores, predominantemente, não possuem capacidade suficiente para produzir suas próprias sementes e, assim, dependem de fornecedores para fazer a multiplicação. Para tanto, buscam selecionar fornecedores adequados, pois, na maioria dos casos, a qualidade do material produzido na lavoura determinará a qualidade da semente comercializada.

Nessa relação, os compradores fornecem aos agricultores os principais insumos para a produção das sementes e a assistência técnica, e fazem adiantamento de pagamento ao agricultor, diminuindo assim o risco de preço, dado que os contratos especificam os valores antecipadamente (Eaton \& Shepherd, 2001). Por outro lado, os compradores exigem conhecimento, habilidade técnica e atendimento a inúmeras especificações para o cumprimento das obrigações (Carvalho \& Nakagawa, 2000; Eaton \& Shepherd, 2001).

O relacionamento entre comprador e fornecedor, fundamentado em confiança, comunicação, adaptação, dependência e satisfação, associados à necessidade de adoção de práticas de qualidade, consiste em fatores importantes para o relacionamento entre agricultores e indústria produtora de sementes, uma vez que relacionamentos colaborativos e duradouros podem trazer inúmeros benefícios para as partes (Ganesan, 1994; Fynes \& Voss, 2002; Dellana \& Kros, 2014; Zhong et al., 2016; Yu \& Huo, 2018).

Destarte, o objetivo deste trabalho foi analisar os fatores de relacionamento e as práticas de gestão da qualidade que caracterizam a interação entre os agricultores multiplicadores de semente de milho (fornecedores) e a indústria produtora de sementes (compradores). Neste sentido, levantar e compreender os fatores de relacionamento existentes na relação entre fornecedores e compradores, que são determinantes para o sucesso da parceria, foram o foco da pesquisa.

Existe vasta literatura mostrando a importância da gestão da qualidade e dos relacionamentos bem-sucedidos em diversos setores da economia. No entanto, grande parte desses estudos aborda tais assuntos de forma isolada, em que a interação entre gestão da qualidade e fatores relacionais é limitada (Mellat-Parast, 2013; Truong et al., 2017). Diante disto, a lacuna de pesquisa a ser explorada consiste em explorar o relacionamento e gestão da qualidade entre comprador e fornecedor de forma integrada. Para preencher essa lacuna, propôs-se compreender a percepção dos fornecedores com relação às suas práticas de qualidade e aos seus relacionamentos. 


\section{Referencial teórico}

Um relacionamento pode ser caracterizado pela interação de indivíduos de diferentes organizações, áreas e níveis hierárquicos, que se envolvem em interações entre empresas, usualmente identificados como comprador e fornecedor (Hakansson, 1982). De fato, as empresas podem ter menor chance de serem competitivas sem a parceria de seus fornecedores (Lummus \& Vokurka, 1999). Uma estratégia da cadeia de suprimentos "orientada para o relacionamento é claramente a melhor escolha para aqueles que desejam prosperar no ambiente global competitivo" (Benton \& Maloni, 2005, p. 19).

A flexibilidade de resposta rápida às mudanças do mercado contribui para 0 desenvolvimento de parcerias (Ziggers \& Trienekens, 1999). A escolha de parceiros adequados contribui para auxiliar a cadeia de suprimentos a criar valor superior para os clientes finais frente à concorrência (Sila et al., 2006).

As empresas passam a desenvolver relacionamentos colaborativos com a finalidade de adquirir recursos e habilidades que não dispõem internamente, visando obter eficiência, flexibilidade e vantagem competitiva (Nyaga et al., 2010). Logo, a qualidade do relacionamento entre comprador e fornecedor estabelece a continuidade do relacionamento (Crosby et al., 1990).

Quando as empresas notaram que podiam ampliar sua competitividade no mercado global ao adotar parcerias, migraram de relacionamentos adversários para cooperativos (Tan et al., 1998). Fatores como comunicação, confiança, adaptação, compromisso, interdependência, satisfação e cooperação são indicadores da força do relacionamento (Fynes \& Voss, 2002).

Para Morgan \& Hunt (1994), o compromisso e a confiança são fundamentais em um relacionamento, pois incentivam os parceiros a promover a preservação da cooperação com os parceiros de troca, recusar parcerias alternativas de curto prazo, em favor dos benefícios esperados em longo prazo obtidos com os parceiros existentes, e considerar ações de alto risco como sendo prudentes, pelo fato de se acreditar que os parceiros não são oportunistas.

Ganesan (1994) ressalta a importância da confiança como determinante dos relacionamentos bem-sucedidos. E acrescenta, ainda, que a dependência e a confiança são fatores decisivos para a qualidade do relacionamento e, consequentemente, para a orientação em longo prazo.

Benton \& Maloni (2005) avaliam como a relação comprador-fornecedor afeta a satisfação dos fornecedores e estabelecem, como elementos críticos de um relacionamento compradorfornecedor, a cooperação, o compromisso, a confiança, a conformidade e a resolução de conflitos. Concluíram que os fornecedores concentram maior atenção em manter o relacionamento com seus compradores, em lugar de seu próprio desempenho.

Mulyo Aji (2016) realizou um estudo com agricultores, com o intuito de avaliar até que ponto os fatores de relacionamentos comprador-fornecedor influenciam a decisão dos agricultores. A conclusão foi que as relações entre ambos foram estabelecidas com base em altos níveis de satisfação, confiança e compromisso em longo prazo, sendo que a qualidade dos produtos e dos serviços ofertados, em vez da entrega e do preço, melhorou significativamente a satisfação dos relacionamentos dos agricultores.

Tsanos \& Zografos (2016) também mostraram que o comportamento colaborativo, baseado em mutualidade, reciprocidade, compromisso e confiança entre parceiros, nas relações de troca, leva a uma maior integração e desempenho da cadeia de suprimentos.

Ambrose et al. (2010) mostraram que compradores e fornecedores têm percepções significativamente diferentes dos elementos que compõem as relações compradorfornecedor. Segundo eles, na visão dos compradores, o compromisso, a confiança, a comunicação e a dependência são fatores ausentes para o desempenho do relacionamento. Para os fornecedores, a confiança, a adaptação e a dependência não são significantes. Por fim, os autores concluem que a confiança e o compromisso são vitais para o sucesso de um relacionamento. 
Resultados semelhantes foram encontrados no estudo de Nyaga et al. (2010), que, ao analisarem a relação entre comprador e fornecedor, mostraram que relacionamentos colaborativos causam satisfação em ambas as partes, sendo que qualquer iniciativa que promova o comprotimento e a confiança resulta em grandes benefícios para 0 relacionamento. Concluem também que apesar de algumas diferenças de ponto de vista, tanto compradores quanto fornecedores têm maiores perspectivas semelhantes no relacionamento do que diferentes quanto a confiança, comprometimento, satisfação, compartilhamento de informações, esforço conjunto e desempenho.

Os trabalhos supramencionados demonstram que várias dimensões/fatores ou interações (confiança, comunicação, adaptação, dependência e satisfação) complementamse e estão fortemente correlacionados como fatores críticos de sucesso para um relacionamento colaborativo na relação comprador-fornecedor. Esses achados de pesquisa também motivaram e instigaram a presente pesquisa.

\section{Metodologia}

Quanto à natureza, esta pesquisa caracteriza-se como aplicada. Com relação aos objetivos, como exploratória e descritiva. No que se refere à forma de abordagem do problema, a pesquisa foi classificada como quali-quantitativa. Quanto aos procedimentos técnicos, foi utilizado uma Survey que tem como propósito testar hipóteses, bem como indicar a existência de associação entre variáveis.

Minas Gerais é o estado brasileiro com a maior produção de sementes de milho do país, seguido pelo estado de Goiás. Essa representatividade deve-se especialmente aos municípios de Paracatu-MG, Unaí-MG e Cristalina-GO, por apresentarem grandes vantagens para a produção de sementes, tais como fatores edafoclimáticos favoráveis e grande concentração de áreas irrigadas por pivô central. Os campos de produção de sementes nessas regiões são dominados pelas principais multinacionais, dentre as quais: Monsanto (recentemente adquirida pela Bayer), Dow Agrosciences, DuPont Pionner, Limagrain, Nidera, Riber Sementes, Helix Sementes, Geneze Sementes e outras de menor porte.

A população-alvo compreendeu agricultores que realizam a multiplicação de sementes de milho para uma dessas multinacionais (indústria produtora de sementes), localizados em Paracatu, Unaí e Guarda-Mor. Foi considerado também o município de Cristalina, no estado de Goiás, mas que faz fronteira com os municípios supracitados. Os critérios de seleção adotados para escolha da população-alvo foram: i) os campos de produção de sementes de milho deveriam estar localizados nas regiões supramencionadas; ii) os produtores deveriam estar com contrato renovado com a indústria produtora de sementes ou deveriam ter algum vínculo contratual durante os dois últimos anos.

Com a finalidade de levantar o maior número de respondentes, foi necessário o contato com a indústria produtora de sementes e com outros profissionais envolvidos do setor. Foram identificados 175 agricultores com esse perfil. Para o desenvolvimento da Survey, foi utilizado como instrumento o questionário estruturado para fazer o levantamento dos dados. O questionário foi elaborado a partir de itens de medição validados em estudos anteriores, os quais foram adaptados para o contexto desta pesquisa, que contém 45 itens, correspondendo a 11 construtos e 10 itens de caráter descritivo.

Os construtos escolhidos para o estudo foram: confiança, comprometimento e satisfação (Benton \& Maloni, 2005); comunicação e adaptação (Heide \& John, 1992); dependência e relacionamento em longo prazo (Ganesan, 1994); atendimentos às especificações (Benton \& Maloni, 2005; Morita \& Flynn, 1997); comprometimento da alta gerência e foco no comprador, e melhoria contínua (Morita \& Flynn, 1997). Todas as medidas utilizaram uma escala Likert que variava entre 1 (discordo totalmente) e 5 (concordo totalmente).

Depois do pré-teste, o instrumento foi transformado em duas versões, física (impressa) e on-line, sendo aplicada aquela que fosse mais conveniente para o respondente, mediante consulta prévia. Após o questionário estar apto para ser aplicado, realizou-se um segundo contato com os profissionais da indústria de sementes com a finalidade de obter uma lista 
contendo o nome e o contato dos seus fornecedores. Ademais, alguns contatos foram sendo informados por outros profissionais ligados ao setor de sementes (revenda de insumos agrícolas) e outros foram fornecidos pelos próprios respondentes da pesquisa. À medida que os contatos foram sendo obtidos, realizava-se um processo de filtragem dos contatos para eliminar e/ou evitar redundâncias.

Ao final, foram identificados e enviados 175 questionários, tendo o retorno de 69. Os questionários recebidos e considerados válidos foram qualificados/direcionados para análise confirmatória e para o teste de hipóteses.

\subsection{Análise fatorial confirmatória e análise de trilhas}

Foram realizadas Análises Fatoriais Confirmatórias (AFCs), conforme proposto por Everitt \& Hothorn (2011). Foram realizadas também análises de trilhas (path analysis) para se testarem as principais hipóteses do estudo (Loehlin, 1998). A análise de trilhas é vista como um caso especial de Modelagem por Equações Estruturais (MEE) (Everitt \& Hothorn, 2011), quando se usam apenas variáveis observadas. Tal estratégia foi preferida ao uso tradicional de MEE pelo tamanho da amostra, usando escores Likert de cada escala como estimativas de escores para cada fator. As análises foram conduzidas no software estatístico R.

O levamento bibliográfico sustentou o desenvolvimento de um modelo conceitual, apresentado na Figura 1, objetivando testar a influência entre os construtos na relação entre a indústria produtora de sementes e os agricultores: fatores relacionais (confiança, comunicação, adaptação, dependência e satisfação), parceria bem-sucedida (comprometimento e orientação em longo prazo do fornecedor) e práticas de qualidade do fornecedor (compromisso da alta gerência, foco no comprador, atendimento às especificações e melhoria contínua).

\begin{tabular}{|c|c|c|c|c|}
\hline $\begin{array}{c}\text { FATORES } \\
\text { RELACIONAIS } \\
\text { (confiança, comunicação, } \\
\text { dependência, satisfação e } \\
\text { adaptação) }\end{array}$ & $\begin{array}{l}\mathrm{H} 1 \mathrm{a} / \mathrm{b} \\
\mathrm{H} 2 \mathrm{a} / \mathrm{b} \\
\mathrm{H} 3 \mathrm{a} / \mathrm{b} \\
\mathrm{H} 4 \mathrm{a} / \mathrm{b} \\
\mathrm{H} 5 \mathrm{a} / \mathrm{b}\end{array}$ & $\begin{array}{l}\text { RELACIONAMENTO } \\
\text { BEM-SUCEDIDO } \\
\text { (comprometimento e } \\
\text { orientação a longo prazo) }\end{array}$ & $\begin{array}{l}\mathrm{H} 6 \\
\mathrm{H} 7\end{array}$ & $\begin{array}{c}\text { PRÁTICAS DE } \\
\text { GESTÃO DA } \\
\text { QUALIDADE } \\
\text { QUALIDso da alta } \\
\text { (compromisso } \\
\text { gerência, foco no } \\
\text { comprador, melhoria } \\
\text { contínua e atendimento às } \\
\text { especificações) }\end{array}$ \\
\hline
\end{tabular}

Figura 1 - Modelo conceitual. Fonte: Elaboração própria.

Assim, as seguintes hipóteses foram testadas:

H1a: O nível de confiança do agricultor influencia positivamente sua orientação em longo prazo.

H1b: O nível de confiança do agricultor influencia positivamente seu comprometimento com o relacionamento.

H2a: A comunicação influencia positivamente a orientação de longo prazo do agricultor.

H2b: A comunicação influencia positivamente o comprometimento do agricultor com o relacionamento.

H3a: A dependência do agricultor influencia positivamente sua orientação de longo prazo.

H3b: A dependência do agricultor influencia positivamente seu comprometimento com o relacionamento.

H4a: O nível de satisfação do agricultor influencia positivamente sua orientação de longo prazo.

H4b: O nível de satisfação do agricultor influencia positivamente seu comprometimento com o relacionamento.

H5a: As adaptações realizadas pelo agricultor influenciam positivamente sua orientação de longo prazo. 
H5b: As adaptações realizadas pelo fornecedor influenciam positivamente seu comprometimento com o relacionamento.

H6: A orientação de longo prazo do agricultor influencia positivamente suas práticas de gerenciamento da qualidade.

H7: O comprometimento do agricultor com o relacionamento influencia positivamente suas práticas de gerenciamento da qualidade.

Levantadas as hipóteses, passa-se para a fase da análise, apresentada a seguir.

\section{Resultados e discussão}

\subsection{Análise fatorial confirmatória (AFC)}

Testaram-se, inicialmente, os pressupostos da AFC sobre normalidades uni e multivariada, e a presença de outliers uni e multivariados. Identificou-se tanto a presença de outliers quanto a falta de normalidade. Por esse motivo, foi utilizado o método de estimação Weighted Least Squares Mean and Variance adjusted (WLSMV) ou Quadrados Mínimos Ponderados Robustos (Muthén et al., 1997), o qual apresenta robustez tanto na quebra de normalidade das distribuições quanto na presença de outliers.

Visando encontrar um modelo de melhor ajuste, de modo a não comprometer o teste da teoria (Hair et al., 2009), foram realizados alguns testes alternativos, inclusive unindo escalas que apresentavam itens com semelhanças semânticas. Assim, a configuração ideal das escalas foi testada a partir do ajuste de cinco modelos alternativos, conforme apresentado na Tabela 1, sendo: i) um modelo com todos os itens de todas as escalas (teórico); ii) um modelo com todos os itens, mas unindo as escalas de melhoria contínua (MC) e de atendimento às especificações (AE) em um único fator; iii) um modelo com todos os itens, mas unindo as escalas de relacionamento em longo prazo (RLP) e comprometimento (Comp) em um único fator; iv) um modelo com todos os itens, mas unindo as escalas de melhoria contínua (MC) e atendimento às especificações (AE) em um único fator, e relacionamento em longo prazo (RLP) e comprometimento (Comp), também em um único fator; v) um modelo com um fator;

Foram utilizados diversos indicadores de ajuste para escolher o melhor modelo (Pilati \& Laros, 2007): Chi-square $\left(\mathrm{X}^{2}\right)$; comparative fit index (CFI); Tucker-Lewis index (TLI); normalized fit index (NFI); standardized root mean square residual (SRMR), e root mean square error of approximation (RMSEA). Esses índices foram divididos conforme proposto por Hair et al. (2009) e Hooper et al. (2008) em: i) medidas de ajuste absoluto ( $X^{2}$, RMSEA e SRMR) com a finalidade de verificar o ajuste global do modelo; ii) medidas de ajuste incremental (NFI, TLI e CFI) para avaliar o ajuste do modelo comparado com um modelo nulo; iii) medidas de ajuste parcimonioso ( $X^{2} / g$.l - qui-quadrado normalizado) que tem como objetivo relacionar a qualidade do ajuste do modelo e o número de coeficientes estimados necessários para atingir tal nível de ajuste.

Tabela 1 - Resultados dos modelos alternativos testados por meio de AFC

\begin{tabular}{|c|c|c|c|c|c|c|c|c|c|}
\hline Modelos testados & $\chi^{2}$ & gl & $\mathbf{p}$ & $\chi^{2} / g 1$ & NFI & CFI & TLI & SRMR & RMSEA \\
\hline $\begin{array}{l}\text { i) Modelo de } 11 \\
\text { fatores (teórico) }\end{array}$ & 807,29 & 764 & 0,134 & 1,057 & 0,876 & 0,896 & 0,883 & 0,102 & 0,028 \\
\hline $\begin{array}{l}\text { ii) Modelo de } 10 \\
\text { fatores (MC + } A E \text { ) }\end{array}$ & 818,89 & 774 & 0,127 & 1,058 & 0,874 & 0,893 & 0,881 & 0,102 & 0,029 \\
\hline $\begin{array}{l}\text { iii) Modelo de } 10 \\
\text { fatores (RLP + COMP) }\end{array}$ & 820,31 & 774 & 0,120 & 1,060 & 0,870 & 0,889 & 0,877 & 0,104 & 0,029 \\
\hline $\begin{array}{l}\text { iv) Modelo de } 9 \text { fatores } \\
\text { (RLP + COMP e MC + } \\
\text { AE) }\end{array}$ & 830,82 & 783 & 0,115 & 1,061 & 0,869 & 0,886 & 0,875 & 0,105 & 0,030 \\
\hline v) Modelo de 1 fator & 901,08 & 819 & 0,023 & 1,100 & 0,815 & 0,804 & 0,794 & 0,127 & 0,038 \\
\hline
\end{tabular}


Os resultados permitem concluir que o modelo teórico (i) [em negrito] para a escala originalmente proposta é aquele que apresenta melhor ajuste aos dados. O modelo de um único fator ( $v$ ) é claramente o pior, enquanto os modelos propostos a partir da união de fatores iniciais (ii, iii e iv) são intermediários. Partindo-se da premissa de que para a avaliação da qualidade de ajuste de um modelo é necessário utilizar diversos índices de ajuste, detalhase a seguir o valor de cada índice encontrado no modelo considerado como o mais adequado, o modelo (i), com o valor de referência sugerido na literatura, divididos como mencionado anteriormente em medidas de ajuste absoluto, de ajuste incremental e de parcimônia.

Inicialmente, a respeito dos índices de ajuste absoluto, o valor qui-quadrado $\left(\chi^{2}\right)$ tem a função de verificar o ajuste global do modelo, de modo a verificar se a diferença entre a matriz prevista e a real será não-significativa $(p>0,05)$ (Hair et al., 2009). Observa-se que o $\chi^{2}$ possui um nível de significância de 0,134, sendo, portanto, não significativo, indicando um ajuste aceitável do modelo (Hair et al., 2009; Pilati \& Laros, 2007). No entanto, conforme os autores, o $\chi^{2}$ é sensível ao tamanho da amostra, o que torna necessário a inclusão de medidas adicionais de ajuste, como, por exemplo, índices RMSEA (raiz do erro quadrático médio aproximado) e SRMR (raiz quadrada média residual).

No caso do RMSEA, um valor menor que 0,08 é desejável (Pilati \& Laros, 2007) e um valor inferior a 0,03 representa um ajuste excelente (Hooper et al., 2008). Logo, no modelo apresentado, tem-se um valor de 0,028, o qual pode ser considerado como um ótimo ajuste.

Para o SRMR, que corresponde à raiz quadrada da diferença entre os resíduos da matriz de covariância da amostra e o modelo de covariância hipotetizado, valores próximos a 0 (zero) e menores que 0,08 indicam que o modelo está bem ajustado (Hooper et al., 2008). Foi encontrado um índice de 0,102, o que representa um ajustamento fraco de acordo com alguns pontos de corte identificados na literatura.

Com relação aos índices de ajuste incremental - NFI (índice de ajuste normalizado), CFI (índice comparativo de ajuste) e TLI (índice de ajustamento Tucker-Lewis) -, esperam-se valores entre 0,90 e 0,95 considerados como ajuste suficiente (Pilati \& Laros, 2007). Logo, obteve-se um valor de 0,876 para NFI, 0,896 para CFI e 0,883 para TLI, todas as medidas de ajuste incremental se aproximam de um ajuste considerado como aceitável.

Por fim, no que se refere à medida de ajuste parcimonioso, considerando o quiquadrado normalizado $\left(\chi^{2} / \mathrm{gl}\right)$, tem-se um valor de 1,057. Este valor encontra-se satisfatório entre os limites recomendados de 1,0 a 2,0 (Hair et al., 2009).

Contudo, este estudo utiliza sete índices para analisar o ajuste do modelo, sendo que o emprego de três ou quatro índices já seriam suficientes para fornecer evidência adequada de ajuste do modelo, desde que utilizado ao menos um índice incremental e um índice absoluto, alem do valor $\chi^{2}$ e dos graus de liberdade associados (Hair et al., 2009). Em síntese, conclui-se que o modelo com 11 fatores (i) foi considerado como o mais adequado, pois apresenta os seguintes valores de índices de ajuste: $\chi^{2}(764)=807,29(p>0,05) ; \mathrm{NFI}=0,876 ; \mathrm{CFI}=0,896$; TLI $=0,883$; SRMR $=0,102$ e RMSEA $=0,028$. Logo, os índices de ajuste qui-quadrado $\left(\chi^{2}\right)$, quiquadrado normalizado $\left(\chi^{2} / g l\right)$ e RMSEA atendem aos critérios para um bom ajustamento do modelo. Os índices NFI, CFI e TLI, com exceção do SRMR, se aproximam dos valores considerados aceitáveis na literatura (Pilati \& Laros, 2007; Hooper et al., 2008; Hair et al., 2009).

A Tabela 2 apresenta as cargas fatoriais e os valores de confiabilidade da solução tida como a mais adequada. De acordo com Hair et al. (2009), a confiabilidade é a capacidade de um conjunto de itens produzir resultados semelhantes sob condições consistentes. O Alfa de Cronbach, em particular, é estimado pela correlação média de um conjunto de itens, sendo uma estimativa mais conservadora da confiabilidade de um teste psicométrico (Cho, 2016). Assim, a medida de confiabilidade varia de 0 a 1, sendo os valores de 0,60 a 0,70 considerados o limite inferior de aceitabilidade (Hair et al., 2009). 
Tabela 2 - Cargas fatoriais e valores de Alfa de Cronbach, como medida de confiabilidade, para o modelo considerado como ideal a partir da AFC

\begin{tabular}{|c|c|c|c|c|c|c|c|c|c|c|c|}
\hline \multirow{2}{*}{ Itens } & \multicolumn{11}{|c|}{ Fatores } \\
\hline & Comu & Adap & Dep & Satisf & Conf & Comp & RLP & FC & AE & MC & CAG \\
\hline comu1 & 0,67 & & & & & & & & & & \\
\hline comu2 & 0,66 & & & & & & & & & & \\
\hline comu3 & 0,55 & & & & & & & & & & \\
\hline adap1 & & 0,80 & & & & & & & & & \\
\hline adap3 & & 0,62 & & & & & & & & & \\
\hline dep1 & & & 0,31 & & & & & & & & \\
\hline dep2 & & & 0,94 & & & & & & & & \\
\hline dep3 & & & 0,46 & & & & & & & & \\
\hline satisf1 & & & & 0,42 & & & & & & & \\
\hline satisf2 & & & & 0,78 & & & & & & & \\
\hline satisf3 & & & & 0,80 & & & & & & & \\
\hline satisf4 & & & & 0,68 & & & & & & & \\
\hline conf1 & & & & & 0,52 & & & & & & \\
\hline conf2 & & & & & 0,63 & & & & & & \\
\hline conf3 & & & & & 0,68 & & & & & & \\
\hline conf4 & & & & & 0,89 & & & & & & \\
\hline conf5 & & & & & 0,65 & & & & & & \\
\hline comp1 & & & & & & 0,56 & & & & & \\
\hline comp2 & & & & & & 0,70 & & & & & \\
\hline comp3 & & & & & & 0,62 & & & & & \\
\hline rlp1 & & & & & & & 0,66 & & & & \\
\hline rlp2 & & & & & & & 0,73 & & & & \\
\hline rlp3 & & & & & & & 0,85 & & & & \\
\hline rlp4 & & & & & & & 0,85 & & & & \\
\hline fc1 & & & & & & & & 0,53 & & & \\
\hline fc2 & & & & & & & & 0,44 & & & \\
\hline fc3 & & & & & & & & 0,66 & & & \\
\hline fc4 & & & & & & & & 0,80 & & & \\
\hline $\mathrm{fc5}$ & & & & & & & & 0,80 & & & \\
\hline atend1 & & & & & & & & & 0,34 & & \\
\hline atend2 & & & & & & & & & 0,47 & & \\
\hline atend3 & & & & & & & & & 0,64 & & \\
\hline atend5 & & & & & & & & & 0,60 & & \\
\hline atend6 & & & & & & & & & 0,66 & & \\
\hline mc1 & & & & & & & & & & 0,59 & \\
\hline mc2 & & & & & & & & & & 0,73 & \\
\hline mc3 & & & & & & & & & & 0,96 & \\
\hline mc4 & & & & & & & & & & 0,94 & \\
\hline cag2 & & & & & & & & & & & 0,43 \\
\hline cag3 & & & & & & & & & & & 0,71 \\
\hline cag4 & & & & & & & & & & & 0,38 \\
\hline cag5 & & & & & & & & & & & 0,70 \\
\hline $\begin{array}{l}\text { Alfa de } \\
\text { Cronbach }\end{array}$ & 0,66 & 0,64 & 0,61 & 0,75 & 0,80 & 0,64 & 0,87 & 0,79 & 0,75 & 0,87 & 0,65 \\
\hline
\end{tabular}

Nota: Identificação dos fatores da escala: Comu = Comunicação; Adap = Adaptação; Dep = Dependência; Satisf = Satisfação do relacionamento; Conf = Confiança; Comp = Comprometimento; RLP = Relacionamento em longo prazo; $\mathrm{FC}=$ Foco no Comprador; $\mathrm{AE}=$ Atendimento às Especificações; $\mathrm{MC}=$ Melhoria contínua; $\mathrm{CAG}=$ Compromisso da Alta Gerência. Fonte: Elaboração própria. 
Observam-se os alfas para os sete construtos de relacionamento, que mantiveram níveis alfa aceitáveis, com comunicação em 0,66, adaptação em 0,64, dependência em 0,61, satisfação em 0,75 e confiança em 0,80 . Em seguida, duas medidas de um relacionamento bem-sucedido, fundamentados no comprometimento e na orientação em longo prazo, também com níveis aceitáveis em 0,64 e 0,87, respectivamente. Finalmente, quatro medidas de práticas de qualidade (foco no comprador, atendimento às especificações, melhoria contínua e compromisso da alta gerência) com valores alfa 0,79, 075, 0,87 e 0,65, respectivamente.

\subsection{Teste de hipóteses e resultados}

A partir dos resultados da AFC, usando o modelo com o melhor ajuste, testaram-se as hipóteses principais do estudo por meio de uma análise de trilhas. A significância das hipóteses foi testada usando estatística $z$, com estimativas de beta consideradas significativamente diferentes de zero quando $z>1,96(p<0,05)$.

Os resultados dos testes de hipóteses são apresentados nas Tabelas 3 e 4 . Primeiramente, apresentam-se os resultados do teste das hipóteses para os fatores relacionais, conforme demonstra a Tabela 3.

Tabela 3 - Hipóteses aceitas ou rejeitadas a partir dos coeficientes de regressão dos fatores de relacionamento, recuperados por meio de uma análise de trilhas

\begin{tabular}{|c|c|c|c|c|c|c|}
\hline Hipóteses & $\beta$ & EP & Valor $p$ & $\begin{array}{l}\text { Sinal } \\
\text { Esperado }\end{array}$ & $\begin{array}{c}\text { Sinal } \\
\text { Observado }\end{array}$ & Conclusão \\
\hline \multicolumn{7}{|l|}{$\begin{array}{l}\text { H1: O nível de confiança do } \\
\text { agricultor influencia positivamente. }\end{array}$} \\
\hline H1a: sua orientação de longo prazo & $-0,01^{\text {NS }}$ & 0,12 & 0,941 & + & Nulo & $\begin{array}{l}\text { Hipótese } \\
\text { rejeitada }\end{array}$ \\
\hline H1b: seu comprometimento & 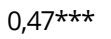 & 0,14 & 0,001 & + & + & Hipótese aceita \\
\hline \multicolumn{7}{|l|}{$\begin{array}{l}\text { H2: O nível de comunicação do } \\
\text { agricultor influencia positivamente. }\end{array}$} \\
\hline H2a: sua orientação de longo prazo & $0,30 * \star$ & 0,10 & 0,002 & + & + & Hipótese aceita \\
\hline H2a: seu comprometimento & $0,26 * \star$ & 0,10 & 0,007 & + & + & Hipótese aceita \\
\hline \multicolumn{7}{|l|}{$\begin{array}{l}\text { H3: A dependência do agricultor } \\
\text { influencia positivamente. }\end{array}$} \\
\hline H3a: sua orientação de longo prazo & $0,20 *$ & 0,10 & 0,050 & + & + & Hipótese aceita \\
\hline H3b: seu comprometimento & $-0,01$ NS & 0,08 & 0,860 & + & Nulo & $\begin{array}{l}\text { Hipótese } \\
\text { rejeitada }\end{array}$ \\
\hline \multicolumn{7}{|l|}{$\begin{array}{l}\text { H4: O nível de satisfação do } \\
\text { agricultor influencia positivamente. }\end{array}$} \\
\hline H4a: sua orientação de longo prazo & $0,44^{* * *}$ & 0,13 & 0,001 & + & + & Hipótese aceita \\
\hline H4b: seu comprometimento & $0,30 *$ & 0,13 & 0,017 & + & + & Hipótese aceita \\
\hline \multicolumn{7}{|l|}{$\begin{array}{l}\text { H5: As adaptações realizadas pelo } \\
\text { agricultor influenciam } \\
\text { positivamente. }\end{array}$} \\
\hline H5a: sua orientação de longo prazo & $0,14^{\mathrm{NS}}$ & 0,10 & 0,177 & + & Nulo & $\begin{array}{l}\text { Hipótese } \\
\text { rejeitada }\end{array}$ \\
\hline H5b: seu comprometimento & $-0,24 * *$ & 0,08 & 0,003 & + & - & $\begin{array}{l}\text { Hipótese } \\
\text { rejeitada }\end{array}$ \\
\hline Variável predita & RLP & \multicolumn{2}{|c|}{ Comp } & & & \\
\hline $\mathrm{R}^{2}$ & 0,573 & \multicolumn{2}{|c|}{0,763} & & & \\
\hline Total de hipóteses aceitas & 6 & & & & & \\
\hline Total de hipóteses rejeitadas & 4 & & & & & \\
\hline
\end{tabular}

Fonte: Elaboração própria. * Estimativa significante a $p<0,05$. ** Estimativa significante a $p<0,01$. *ᄎ* Estimativa significante a $p<0,001$. NS: Não significativo. 
Foi hipotetizado que o nível de confiança do agricultor influencia positivamente sua orientação em longo prazo (H1a). No entanto, essa hipótese foi rejeitada, na medida em que não foi constatada nenhuma influência significativa da confiança no desejo do fornecedor em permanecer no relacionamento, pois, conforme apresentado, o coeficiente padronizado $(\beta)$ de 0,01 não foi significativo estatisticamente $(p>0,05)$. Por outro lado, a confiança demonstrou influenciar fortemente o comprometimento $(\beta=047, p<0,001)$ dos agricultores em seu relacionamento com a indústria de sementes, apoiando $\mathrm{H} 1 \mathrm{~b}$.

A segunda hipótese refere-se à comunicação na relação agricultor-indústria. Para tanto, a comunicação foi hipotetizada para impactar positivamente tanto a orientação em longo prazo ( $\mathrm{H} 2 \mathrm{a})$ quanto o comprometimento do agricultor $(\mathrm{H} 2 \mathrm{~b})$. Ambas as hipóteses foram aceitas. Logo, pode-se dizer que a comunicação influencia positivamente o desejo do agricultor em manter um relacionamento de longo prazo com sua principal compradora $(\beta=$ $0,30, p<0,01)$, assim como também influencia significativamente o seu comprometimento com o relacionamento $(\beta=0,26, p<0,01)$.

Em seguida, a hipótese ( $\mathrm{H} 3 \mathrm{a}$ ) de que o nível de dependência do agricultor influencia positivamente a orientação em longo prazo é suportada, com efeito marginalmente significativo $(\beta=0,20, p=0,05)$. Em contraposição, a hipótese $(\mathrm{H} 3 \mathrm{~b})$ de que o nível de dependência influencia positivamente o comprometimento não foi significativa $(\beta=-0,01, p>$ 0,05), sendo, portanto, rejeitada.

Adicionalmente, a satisfação foi hipotetizada por influenciar positivamente a orientação em longo prazo ( $\mathrm{H} 4 \mathrm{a})$ e o comprometimento (H4b). Ambas as hipóteses foram apoiadas na perspectiva do agricultor. Com isso, a satisfação apresentou uma influência positiva e altamente significativa $(\beta=0,44, p<0,001)$ no desejo do agricultor em desenvolver um relacionamento de longo prazo com sua principal indústria de sementes. Assim, também, o nível de satisfação do agricultor mostrou um efeito significativo e positivo no comprometimento $(\beta=0,30, p<0,05)$, fornecendo um suporte moderado para $\mathrm{H} 4 \mathrm{~b}$.

Por fim, ainda quanto aos aspectos relacionais, foi hipotetizado que as adaptações realizadas pelo agricultor em seu sistema de produção agrícola impactariam positivamente a sua orientação em longo prazo (H5a) e o comprometimento (H5b). Embora as adaptações não apresentem uma influência significativa na orientação em longo prazo $(\beta=0,14, p>0,05)$, o estudo encontrou uma influencia significativa negativa $(\beta=-0,24, p<0,01)$ das adaptações no comprometimento do agricultor.

O valor de $\mathrm{R}^{2}$ indica que as cinco variáveis preditoras incluídas na equação de regressão (confiança, comunicação, satisfação, adaptação e dependência) explicam 57,3\% da variância na orientação em longo prazo e 76,3\% da variância no comprometimento, o que indica que a presença desses fatores, embora contribuam para uma orientação em longo prazo, está mais voltada para o comprometimento do produtor em benefício do relacionamento.

A Tabela 4 apresenta os resultados das hipóteses aceitas e rejeitadas quanto à influência de um relacionamento bem-sucedido (orientação em longo prazo e comprometimento) nas práticas de gestão da qualidade.

A hipótese $(\mathrm{H} 6)$ postula que o desejo dos agricultores em manter um relacionamento em longo prazo com sua principal compradora influencie positivamente nas práticas de gestão da qualidade. Foi possível constatar que essa hipótese foi aceita. Neste sentido, destaca-se uma expressiva influência da orientação em longo prazo no compromisso do agricultor com a qualidade $(\beta=0,47, p=0,002)$, assim como uma influência positiva e significativa da orientação em longo prazo no foco no comprador $(\beta=0,38, p=0,004)$. O desejo do agricultor em estabelecer um relacionamento duradouro tem uma influência moderada no atendimento às especificações $(\beta=0,32, p=0,031)$ e na melhoria contínua $(\beta=0,36, p=0,018)$.

É esperado que o comprometimento do agricultor influencie positivamente as práticas de gestão da qualidade dos agricultores (H7). Essa hipótese foi parcialmente aceita. Este estudo mostra que o comprometimento influencia positivamente o foco no comprador $(\beta=$ $0,36, p=0,003)$ e o atendimento às especificações $(\beta=0,28, p=0,016)$. Contrariamente, não foi constatada influência significativa do comprometimento no compromisso do agricultor 
com a qualidade $(\beta=-0,13, p>0,05)$, assim como não houve influência significativa do comprometimento do agricultor com a melhoria contínua $(\beta=0,19, p>0,05)$.

Tabela 4 - Hipóteses aceitas ou rejeitadas a partir dos coeficientes de regressão das práticas de gestão da qualidade, recuperados por meio de uma análise de trilhas

\begin{tabular}{|c|c|c|c|c|c|c|}
\hline \multicolumn{7}{|l|}{$\begin{array}{l}\text { H6: A orientação em longo prazo } \\
\text { dos agricultores influencia } \\
\text { positivamente as práticas de } \\
\text { gerenciamento da qualidade. }\end{array}$} \\
\hline Compromisso da Alta Gerência & $0,47^{\star \star}$ & 0,15 & 0,002 & + & + & Hipótese aceita \\
\hline Foco no comprador & $0,38 * *$ & 0,13 & 0,004 & + & + & Hipótese aceita \\
\hline Atendimento às especificações & $0,32 *$ & 0,15 & 0,031 & + & + & Hipótese aceita \\
\hline Melhora contínua & $0,36^{*}$ & 0,15 & 0,018 & + & + & Hipótese aceita \\
\hline \multicolumn{7}{|l|}{$\begin{array}{l}\text { H7: O comprometimento dos } \\
\text { agricultores influencia } \\
\text { positivamente as práticas de } \\
\text { gerenciamento da qualidade. }\end{array}$} \\
\hline Compromisso da Alta Gerência & $-0,13^{\text {NS }}$ & 0,19 & 0,505 & + & Nulo & $\begin{array}{l}\text { Hipótese } \\
\text { rejeitada }\end{array}$ \\
\hline Foco no comprador & $0,36 * \star$ & 0,12 & 0,003 & + & + & Hipótese aceita \\
\hline Atendimento às especificações & $0,28^{*}$ & 0,11 & 0,016 & + & + & Hipótese aceita \\
\hline Melhora contínua & 0,19 NS & 0,13 & 0,142 & + & Nulo & $\begin{array}{l}\text { Hipótese } \\
\text { rejeitada }\end{array}$ \\
\hline Variável predita & CAG & FC & & $\mathrm{AE}$ & MC & \\
\hline $\mathrm{R}^{2}$ & 0,177 & 0,416 & & 0,268 & 0,277 & \\
\hline Total de hipóteses aceitas & 6 & & & & & \\
\hline Total de hipóteses rejeitadas & 2 & & & & & \\
\hline
\end{tabular}

O valor de $\mathrm{R}^{2}$ indica que apenas $16,8 \%$ da variância no compromisso dos agricultores com a qualidade foi explicada pela orientação em longo prazo do relacionamento e pelo comprometimento, enquanto que $41,6 \%$ da variância do foco no comprador foi explicada pela orientação em longo prazo do relacionamento e pelo comprometimento. O comprometimento e a orientação em longo prazo explicam $26,8 \%$ da variância no atendimento às especificações e $27,7 \%$ na melhoria contínua.

Inicialmente, analisando os valores de $\beta$ dos principais fatores de relacionamento fornecidos na Tabela 3, pode-se dizer que a confiança e a satisfação, seguidas da comunicação e da dependência, são os principais atributos relacionais que caracterizam o relacionamento entre os agricultores e a indústria produtora de sementes, na percepção dos produtores rurais. A confiança está mais inclinada a contribuir para o comprometimento dos agricultores, enquanto a satisfação e a comunicação contribuem tanto para o comprometimento quanto para a orientação em longo prazo. A dependência contribui especialmente para que os agricultores desejem manter um relacionamento em longo prazo com a indústria de sementes.

A satisfação do agricultor e a troca de informações entre as partes são fatores relacionais decisivos para uma parceria bem-sucedida entre os agricultores e a indústria produtora de sementes. Destaca-se a presença da confiança para promover o comprometimento dos agricultores com o relacionamento e de uma moderada dependência dos agricultores com a compradora, o que estimula a continuidade do relacionamento.

Os resultados demonstram que o desenvolvimento de uma parceria bem-sucedida, fundamentada no comprometimento e na orientação em longo prazo, promove a gestão da qualidade nos empreendimentos rurais de produção de sementes, especialmente por meio das seguintes práticas de foco no comprador e atendimento às especificações. 


\subsection{Influência da confiança para um relacionamento bem-sucedido}

Corroborando com estudos anteriores (Morgan \& Hunt, 1994; Yang et al., 2008; Nyaga et al., 2010; Tsanos \& Zografos, 2016; Soares et al., 2017), foi comprovado que a confiança dos agricultores tem uma influência forte e positiva no comprometimento ( $\mathrm{H} 1 \mathrm{a})$, mas não há nenhuma influência significativa da confiança na orientação em longo prazo do agricultor (H1b) (Tabela 3). O resultado de (H1b) contradiz os achados de Ganesan (1994), que identificou influência positiva entre a confiança e a orientação em longo prazo.

Estes resultados sugerem que a confiança conduz os agricultores a dedicarem esforços para manter o relacionamento com sua principal compradora de sementes. No entanto, isso não quer dizer que o nível de confiança seja suficiente para que os agricultores desejem manter um relacionamento em longo prazo com sua principal sementeira. Ademais, é claro que isso não impede que a confiança e o comprometimento possam levar futuramente a uma estabilidade do relacionamento (Yang et al., 2008), mas infere-se que existem incertezas quanto à longevidade do relacionamento por parte dos agricultores.

Sugerem-se duas explicações para tal afirmação. A primeira é que a confiança entre as partes pode ser resultante de experiências anteriores (Wang et al., 2015). Assim, talvez haja uma relação inversa, em que o desejo em estabelecer um relacionamento em longo prazo constrói uma expressiva parcela de confiança na relação. A confiança dos agricultores pode ser ampliada conforme o relacionamento vai sendo conduzido, juntamente com sua durabilidade. Ganesan (1994) corrobora com esse argumento quando destaca a necessidade de verificar a ocorrência de uma sequência inversa, em que a orientação em longo prazo afeta a confiança.

A segunda explicação refere-se ao fato de a confiança estar relacionada às questões contratuais entre as partes. Wang et al. (2015) mostram que o nível de confiança na orientação de longo prazo do relacionamento depende do nível de obrigações contratuais estabelecido entre as partes. Segundo os autores, as obrigações contratuais são formas de proteção contra comportamentos oportunistas, além de que os níveis de confiança e o desejo de continuar o relacionamento dependerão exclusivamente da parte que esteja protegida pelas obrigações contratuais. Assim, a confiança dos agricultores em sua principal compradora pode estar relacionada às obrigações contratuais a serem cumpridas exclusivamente por eles, o que, consequentemente, provoca incerteza na vontade de permanecer no relacionamento.

Contrariamente à afirmação de Fynes \& Voss (2002), a confiança na percepção dos agricultores não está sendo suficiente para reduzir as especificações e o monitoramento dos contratos, bem como para fornecer incentivos para a cooperação e reduzir as incertezas do relacionamento. Para tanto, independentemente da confiança, os agricultores entendem que tudo depende do atendimento às expectativas da indústria de sementes, uma vez que os compradores podem buscar agricultores alternativos, o que comprometeria a continuidade do relacionamento. Essa constatação corrobora com os achados de Nyaga et al. (2010).

Neste sentido, o investimento em confiança poderia favorecer o relacionamento agricultor-indústria, sobretudo porque a confiança recíproca é um fator crítico para o sucesso do relacionamento (Spekman, 1988; Toni et al., 1994). Postula-se também sobre a necessidade de a equipe técnica conquistar a confiança do agricultor, facilitando a condução das exigências normativas necessárias para a manutenção de padrões de qualidade e produtividade das operações agrícolas (Eaton \& Shepherd, 2001).

\subsection{Influência da comunicação para um relacionamento bem-sucedido}

Os resultados apontam que a comunicação influencia positivamente 0 comprometimento, semelhantemente aos achados de Nyaga et al. (2010), e também influencia significativamente a orientação em longo prazo dos agricultores. Logo, sugere-se que quanto maior a frequência de troca de informações, maiores as chances de sucesso da parceria. Isso ocorre porque a comunicação desempenha papel fundamental na relação entre os agricultores e os técnicos de campo da indústria de sementes, na medida em que, por ora, 
todas as atividades para a condução do campo de produção de sementes precisam ser transmitidas de alguma forma aos agricultores ou aos seus funcionários. Ademais, a comunicação pode contribuir para a resolução de conflitos que porventura possam surgir entre os agricultores e a indústria de sementes (Ambrose et al., 2010; Mulyo Aji, 2016).

Desse modo, a confirmação de que o nível de troca de informações é importante para os fornecedores é apoiada por estudos anteriores (Anderson \& Narus, 1990; Ambrose et al., 2010; Nyaga et al., 2010).

\subsection{Influência da dependência para um relacionamento bem-sucedido}

Os resultados demonstram que a dependência tem influência fraca no desejo dos agricultores em estabelecer um relacionamento em longo prazo com sua principal compradora ( $\mathrm{H3a}$ ). Por outro lado, assim como comprovado de maneira semelhante por Ambrose et al. (2010), foi constatado que não houve influência significativa da dependência no comprometimento dos fornecedores (H3b).

Esses achados sugerem que a dependência dos agricultores em sua principal indústria de sementes contribui de certa maneira para que os agricultores desejem construir um relacionamento duradouro. No entanto, o nível de dependência pode não ser suficiente para motivar os agricultores a dedicarem esforços extras em beneficio do relacionamento. Apesar das incertezas, os agricultores acreditam na possibilidade de negociar com outra empresa, o que mostra que eles não são totalmente dependentes da sua principal indústria de sementes.

O nível moderado de dependência pode ser explicado por algumas vantagens obtidas pelo agricultor ao estabelecer uma relação comercial com a indústria de sementes, como, por exemplo, receber determinado valor por hectare plantado, independentemente da produtividade, além de ganhar a maior parte dos insumos utilizados na manutenção da lavoura, como defensivos e sementes. Porém, em última circunstância, caso não venham a negociar com outra indústria para a produção de sementes, os agricultores poderão cultivar outro produto agrícola com finalidade comercial em suas propriedades.

Os resultados demonstram que existe certa dependência dos agricultores da indústria de sementes, o que faz com que eles desejem estabelecer um relacionamento em longo prazo (Ganesan, 1994), mas, caso não seja possível, eles podem procurar outras empresas para a mesma finalidade, ou ainda, realizar o cultivo de outras culturas para comercialização.

\subsection{Influência da satisfação para um relacionamento bem-sucedido}

Os resultados apontam que a satisfação do agricultor com sua principal indústria de sementes influencia fortemente a orientação em longo prazo $(\mathrm{H} 4 \mathrm{a})$, assim como postulado por Ganesan (1994) e Wang et al. (2015). A satisfação do agricultor também influencia o comprometimento (H4b).

É importante ressaltar que o nível de satisfação dos agricultores determinará o sucesso da parceria. Desta maneira, como proposto por Benton \& Maloni (2005) e Wang et al. (2015), a satisfação dos agricultores no relacionamento com sua principal indústria de sementes é um fator crítico de sucesso no desenvolvimento de uma parceria bem-sucedida, com base no comprometimento e na orientação em longo prazo.

\subsection{Influência da adaptação para um relacionamento bem-sucedido}

As adaptações (ou investimento em ativos específicos) realizadas pelos agricultores em seus sistemas de produção não foram significativas para influenciar a orientação em longo prazo (H5a). Preconizam-se duas possíveis explicações para a rejeição dessa hipótese. A primeira justifica-se pelo fato de que as adaptações não necessariamente são direcionadas exclusivamente para uma única finalidade. Por exemplo, o investimento em tecnologia agrícola poderá beneficiar tanto a indústria de sementes quanto o próprio produtor, pois ele poderá destinar tais recursos para outras áreas de cultivo em seu empreendimento agrícola. A segunda explicação está ligada com o fato de que as adaptações realizadas pelos 
agricultores podem ter uma influência superior ao desejo em continuar o relacionamento, como, por exemplo, remuneração extra fornecida pela indústria de sementes oriunda das adaptações no sistema agrícola. Contudo, infere-se que as adaptações não são significativas para influenciar o desejo dos agricultores em continuar o relacionamento.

Ao analisar a hipótese ( $\mathrm{H} 5 \mathrm{~b})$, foi demonstrada influência fortemente significativa e negativa entre adaptação e comprometimento. Esse resultado contradiz os achados de Nyaga et al. (2010), que identificaram que o investimento dedicado ao relacionamento traz impacto positivo e significativo no comprometimento. $\mathrm{O}$ resultado de $(\mathrm{H} 5 \mathrm{~b})$ indica que pode haver um efeito inverso, ou seja, tudo dependerá do quão comprometido esteja o agricultor com seu relacionamento para influenciá-lo a realizar as adaptações no sistema de produção.

Portanto, conclui-se que as adaptações não são suficientes para que o agricultor deseje manter um relacionamento em longo prazo, nem para motivá-lo a dedicar empenho para manter o relacionamento.

\subsection{Influência do relacionamento bem-sucedido nas práticas de gestão da qualidade}

$\mathrm{O}$ teste de hipóteses certificou que tanto a orientação em longo prazo $(\mathrm{H} 6)$ quanto o comprometimento dos agricultores com o relacionamento $(\mathrm{H} 7)$ influenciam as práticas de gestão da qualidade. Em outras palavras, a parceria bem-sucedida é forte preditora das práticas de gestão da qualidade dos agricultores. Logo, foi possível verificar que algumas práticas tiveram níveis diferentes de influência.

Discutindo inicialmente (H6), observa-se que a orientação em longo prazo faz com que os produtores estejam engajados com as questões de qualidade. Nesse sentido, o desejo em manter um relacionamento em longo prazo destaca-se por influenciar o compromisso da alta gerência. $O$ desejo dos agricultores em desenvolver um relacionamento duradouro faz com que eles estejam comprometidos com a qualidade, além de transmitir a ideia às demais pessoas envolvidas nas atividades agrícolas, especialmente aos funcionários, de que a qualidade é algo que deve ser priorizada nas operações agrícolas para a produção de sementes.

Uma orientação em longo prazo faz com que os agricultores tenham foco na sua principal compradora e, envolvidos nesse propósito, acredita-se que busquem verificar constantemente as reclamações da indústria produtora de sementes visando ao atendimento de suas expectativas. Não menos importante, foi possível constatar ainda que o desejo por um relacionamento duradouro leve à busca pela melhoria contínua e pelo atendimento às especificações da indústria produtora de sementes.

Por fim, houve a aceitação parcial da hipótese (H7), pois apenas duas práticas de gestão da qualidade foram significativamente influenciadas pelo comprometimento.

Os resultados apontam que o comprometimento dos agricultores com o relacionamento influencia significativamente apenas as práticas: foco no comprador e atendimento às especificações. Isso reforça que o nível de esforço em manter o relacionamento com a indústria de sementes consistirá essencialmente em atender expectativas e especificações da principal compradora. Por outro lado, o comprometimento não exerceu nenhuma influência significativa no compromisso da alta gerência e na melhoria contínua. Esse fato pode ser justificado porque os fornecedores podem perceber que os compradores não necessariamente retribuem níveis crescentes de comprometimento agregados a novas oportunidades de negócios (Nyaga et al., 2010). Nesse sentido, um relacionamento bemsucedido aliado à certeza de futuras negociações desempenha papel importante para que os agricultores aumentem o compromisso pela qualidade (Lai et al., 2005).

Além disso, o fato de o comprometimento com o relacionamento não influenciar o compromisso da alta gerência está relacionado com os achados de Kaye \& Anderson (1999), que mostraram que a qualidade é vista como sendo responsabilidade da outra parte. Nesse sentido, os agricultores podem não se sentir responsáveis pela qualidade, atribuindo essa responsabilidade à indústria de sementes. 
Ao analisar os valores dos coeficientes $\beta$ da Tabela 4, pode-se dizer que a orientação em longo prazo promove o compromisso dos agricultores com a qualidade $(\beta=0,47)$, foco no comprador $(\beta=0,38)$, melhoria contínua $(\beta=0,36)$ e atendimento às especificações $(\beta=0,36)$.

Já o comprometimento do agricultor com o relacionamento influencia exclusivamente 0 foco no comprador $(\beta=0,36)$ e o atendimento às especificações $(\beta=0,28)$, ou seja, quanto maior o nível de comprometimento dos agricultores em seu relacionamento com sua principal compradora, maiores os esforços dedicados à satisfação e ao atendimento às especificações da indústria de sementes. Portanto, um relacionamento bem-sucedido faz com que os agricultores estejam voltados para a satisfação e para o atendimento das especificações da indústria de sementes quanto aos serviços prestados.

\section{Conclusões}

As informações apresentam a direção e o grau de influência entre cada variável proposta neste estudo. No entanto, tendo em vista que a orientação em longo prazo e o comprometimento foram previstos para influenciarem as práticas de gestão da qualidade adotadas pelos agricultores de sementes de milho, faz-se necessário analisar em que medida essas variáveis preditoras explicam cada prática de gestão da qualidade. Desse modo, utilizando-se os valores $\mathrm{R}^{2}$ apresentados na Tabela 4, pode-se chegar a algumas conclusões.

O valor de $\mathrm{R}^{2}$ é de $41,6 \%$, o que significa que aproximadamente $42 \%$ da variância do foco no comprador foi explicado pelo pela orientação em longo prazo e pelo comprometimento. Assim, um relacionamento bem-sucedido explica uma expressiva quantidade da variação do foco no comprador. Logo, infere-se que um relacionamento bem-sucedido desperta nos agricultores uma preocupação e um interesse em atender às necessidades da indústria de sementes, com a finalidade de aumentar a satisfação com os serviços prestados, assim como foi constatado por (Zhong et al., 2016).

O comprometimento e a orientação em longo prazo representam $27,7 \%$ da variação na melhoria contínua e $26,8 \%$ da variação no atendimento às especificações. Apesar de o relacionamento benéfico explicar aproximadamente $30 \%$ da variação, tanto na melhoria contínua quanto no atendimento às especificações, é evidente que existem $70 \%$ da variação para ambos os construtos que são resultantes de outros aspectos. Neste sentido, os agricultores compreendem a melhoria contínua e o atendimento às especificações como prérequisitos para manterem-se no mercado de produção de sementes, independentemente da natureza do relacionamento entre eles e a indústria, assim como achado por (Fynes \& Voss, 2002).

O comprometimento e a orientação em longo prazo são responsáveis por apenas $17,7 \%$ da variação no comprometimento da alta gerência, indicando que $82,3 \%$ da variação no comprometimento dos agricultores com a qualidade não pode ser explicada apenas pelo relacionamento bem-sucedido, indicando que existem outros fatores a serem considerados.

Se o comprometimento da alta gerência se refere ao reconhecimento pela responsabilidade da qualidade (Zhong et al., 2016), destaca-se uma preocupação quanto ao envolvimento dos agricultores nas questões de qualidade em conjunto com sua principal indústria produtora de sementes. Afinal, um gestor comprometido com a qualidade deve ter a consciência de que a qualidade de todos os parceiros envolvidos na cadeia de suprimentos, inclusive a dele, afetará o resultado da qualidade do produto final (Zhong et al., 2016).

Nesse sentido, chama-se a atenção para as relações bem-sucedidas serem prioridades tanto pelo fornecedor quanto pelo comprador, corroborando com o que foi dito por diversos autores (Benton \& Maloni, 2005; Sila et al., 2006; Dellana \& Kros, 2014; Soares et al., 2017), que reconhecem que, para ampliar o foco pela qualidade, os gestores devem envolver os fornecedores em suas práticas de qualidade, notadamente no desenvolvimento de parcerias com fornecedores (Dellana \& Kros, 2014). Assim, a responsabilidade pela qualidade deve ser assumida e reconhecida por todos os membros da cadeia de suprimentos, inclusive pelos fornecedores. 
A análise multivariada de dados, especialmente o teste de hipóteses sobre a relação comprador-fornecedor, demonstra o nível de influência e a orientação de cada fator relacional (confiança, comunicação, dependência, satisfação e adaptação) para promover um relacionamento bem-sucedido (fundamentado na orientação em longo prazo e o comprometimento), bem como a influência de cada um desses fatores nas práticas de gestão da qualidade.

Identificou-se, na perspectiva dos agricultores, que a satisfação e a comunicação são fatores cruciais para promover um relacionamento bem-sucedido com a indústria de sementes, assim como a confiança, que leva a elevados índices de comprometimento, enquanto que a dependência contribui para o desejo dos agricultores em desenvolver um relacionamento duradouro. Adicionalmente, tem-se que a orientação em longo prazo faz com os agricultores estejam empenhados em adotar práticas de gestão de qualidade, embora tais práticas apresentem níveis diferentes de influência. Por outro lado, o comprometimento conduz, especialmente, para a prática de foco no comprador e o atendimento às especificações.

Adicionalmente, a análise de regressão apresenta uma visão mais ampla da interação de um relacionamento bem-sucedido com cada prática de gestão da qualidade proposta neste estudo, e não restam dúvidas que um relacionamento bem-sucedido entre os agricultores e a indústria produtora de sementes, na percepção dos agricultores, conduz à gestão da qualidade.

Destarte, identificou-se que um relacionamento bem-sucedido contribui especialmente para que os agricultores estejam interessados na satisfação de sua principal compradora. Dessa forma, o relacionamento bem-sucedido também explica uma parcela de outras práticas como a melhoria contínua, o atendimento às especificações e o comprometimento da alta gerência.

Uma descoberta interessante é a possibilidade de existirem fatores que também exerçam influência na adoção das práticas de gestão da qualidade pelos agricultores, especialmente quanto ao comprometimento da alta gerência, à melhoria contínua e ao atendimento às especificações. Há de se ressaltar que esses fatores podem não ser direcionados apenas como responsabilidade dos agricultores, mas também da indústria produtora de sementes. Assim, tanto gestores da indústria quanto os agricultores precisam reconhecer sua responsabilidade pela qualidade.

De fato, o estudo mostra a importância dos relacionamentos estruturados entre as partes envolvidas de se investir na qualidade do relacionamento para promover relações comerciais bem-sucedidas.

Este estudo traz contribuições importantes. Identificou os principais antecedentes relacionais para uma parceria bem-sucedida. Apresentou em que medida um relacionamento bem-sucedido, tendo como base o comprometimento e a orientação em longo prazo, influencia cada prática de gestão da qualidade. Abordou a importância da gestão da qualidade para relações comerciais no agronegócio, mais especificamente as relações do agricultor de sementes e a indústria.

É importante ressaltar que o setor de sementes apresenta características similares às descobertas evidenciadas no contexto industrial e de serviços, abrindo oportunidades de pesquisa que ainda são pouco exploradas no agronegócio, para definitivamente comprovar sua relação e, notadamente, sua aplicação em outras cadeias produtivas do agronegócio.

\section{Referências}

Ambrose, E., Marshall, D., \& Lynch, D. (2010). Buyer supplier perspectives on supply chain relationships. International Journal of Operations \& Production Management, 30(12), 1269-1290.

Anderson, J. C., \& Narus, J. A. (1990). A model of distributor firm and manufacturer firm working partnerships. Journal of Marketing, 54(1), 42-58.

Associação Brasileira de Sementes e Mudas - ABRASEM. (2016). Anuário 2016. Brasília. Recuperado em 29 de julho de 2018, de http://www.abrasem.com.br/anuarios/ 
Benton, W. C., \& Maloni, M. (2005). The influence of power driven buyer/seller relationships on supply chain satisfaction. Journal of Operations Management, 23(1), 1-22.

Bonny, S. (2017). Corporate concentration and technological change in the global seed industry. Sustainability, 9(9), 1632.

Brasil. Companhia Nacional de Abastecimento - CONAB. (2018). Acompanhamento safra brasileira de grãos (Vol. 4, No. 12, Safra 2016/2017, Setembro de 2017). Brasília. Recuperado em 18 de fevereiro de 2018, de http://www.conab.gov.br/conteudos.php?a=1253\&t=

Carvalho, N. M., \& Nakagawa, J. (2000). Sementes: ciência, tecnologia e produção. Campinas: Fundação Cargill.

Cho, E. (2016). Making reliability reliable: a systematic approach to reliability coefficients. Organizational Research Methods, 19(4), 651-682.

Crosby, L. A., Evans, K. R., \& Cowles, D. (1990). Relationship quality in services selling: an interpersonal influence perspective. Journal of Marketing, 54(3), 68-81.

Dellana, S. A., \& Kros, J. F. (2014). An exploration of quality management practices, perceptions and program maturity in the supply chain. International Journal of Operations \& Production Management, 34(6), 786-806.

Dudin, M. N., Smirnova, O. O., Vysotskya, N. V., Frolova, E. E., \& Vikova, N. G. (2015). The deming cycle (PDCA) concept as an efficient tool for continuous quality improvement in the agribusiness. Asian Social Science, 11(1), 239-246.

Eaton, C., \& Shepherd, A. W. (2001). Contract farming: partnerships for growth (Agricultural Services Bulletin). Rome: FAO.

Everitt, B., \& Hothorn, T. (2011). An introduction to applied multivariate analysis with R. New York: Springer.

Fynes, B., \& Voss, C. (2002). The moderating effect of buyer-supllier relationships on quality practices and performance. International Journal of Operations \& Production Management, 22(6), 589-613.

Ganesan, S. (1994). Determinants of long-term in buyer-seller orientation relationships. Journal of Marketing, 58(2), 1-19.

Hair, J. F., Black, W. C., Babin, B. J., Anderson, R. E., \& Tatham, R. L. (2009). Análise multivariada de dados. Porto Alegre: Bookman.

Hakansson, H. (1982). International marketing and purchasing of industrial goods. Chichester: Wiley.

Heide, J. B., \& John, G. (1992). Do norms matter in markerting relationships? Journal of Marketing, 56(2), 32-44.

Hooper, D., Coughlan, J., \& Mullen, M. R. (2008). Structural equation modelling: guidelines for determining model fit. Electronic Journal of Business Research Methods, 6(1), 53-60.

Kaye, M., \& Anderson, R. (1999). Continuous improvement: the ten essential criteria. International Journal of Quality \& Reliability Management, 16(5), 485-509.

Lai, K. H., Cheng, T. C. E., \& Yeung, A. C. L. (2005). Relationship stability and supplier commitment to quality. International Journal of Production Economics, 96(3), 397-410. http://dx.doi.org/10.1016/j.ijpe.2004.07.005

Loehlin, J. C. (1998). Latent variable models: an introduction to factor, path, and structural analysis. Mahwah, NJ: Lawrence Erlbaum Associates, Inc.

Lummus, R. R., \& Vokurka, R. J. (1999). Defining supply chain management: a historical perspective and practical guidelines. Industrial Management \& Data Systems, 99(1), 11-17.

Mellat-Parast, M. (2013). Supply chain quality management: an inter organizational learning perspective. International Journal of Quality \& Reliability Management, 30(5), 511-529.

Morgan, R. M., \& Hunt, S. D. (1994). The commitment-trust theory of relationship marketing. Journal of Marketing, 58(3), 20-38.

Morita, M., \& Flynn, E. J. (1997). The linkage among management systems, practices and behaviour in successful manufacturing strategy. International Journal of Operations \& Production Management, 17(10), 967-993.

Mulyo Aji, J. M. (2016). Exploring farmer-supplier relationships in the East Java seed potato market. Agriculture and Agricultural Science Procedia, 9, 83-94. 
Muthén, B. O., Du Toit, S. H. C., \& Spisic, D. (1997). Robust inference using weighted least squares and quadratic estimating equations in latent variable modeling with categorical and continuous outcomes (Technical report). Los Angeles, CA: University of California.

Nyaga, G. N., Whipple, J. M., \& Lynch, D. F. (2010). Examining supply chain relationships: Do buyer and supplier perspectives on collaborative relationships differ? Journal of Operations Management, 28(2), 101-114.

Pilati, R., \& Laros, J. A. (2007). Modelos de equações estruturais em psicologia: conceitos e aplicações. Psicologia: Teoria e Pesquisa, 23(2), 205-216.

Sila, I., Ebrahimpour, M., \& Birkholz, C. (2006). Quality in supply chains: an empirical analysis. Supply Chain Management, 11(6), 491-502.

Soares, A., Soltani, E., \& Liao, Y.-Y. (2017). The influence of supply chain quality management practices on quality performance: an empirical investigation. Supply Chain Management, 22(2), 122-144.

Spekman, R. E. (1988). Strategic supplier selection: Understanding long-term buyer relationships. Business Horizons, 31(4), 75-81.

Tan, K. C., Handfield, R. B., \& Krause, D. R. (1998). Enhancing the firm's performance through quality and supply base management: an empirical study. International Journal of Production Research, 36(10), 2813-2837.

Toni, A., Nassimbeni, G., \& Tonchia, S. (1994). New trends in the supply environment. Logistics Information Management, 7(4), 41-50.

Truong, H. Q., Sameiro, M., Fernandes, A. C., Sampaio, P., Duong, B. A. T., Duong, H. H., \& Vilhenac, E. (2017). Supply chain management practices and firms' operational performance. International Journal of Quality \& Reliability Management, 34(1), 53-67.

Tsanos, C. S., \& Zografos, K. G. (2016). The effects of behavioural supply chain relationship antecedents on integration and performance. Supply Chain Management, 21(6), 678-693.

Wang, C. L., Shi, Y., \& Barnes, B. R. (2015). The role of satisfaction, trust and contractual obligation on long-term orientation. Journal of Business Research, 68(3), 473-479.

Yang, J., Wang, J., Wong, C., \& Lai, K. (2008). Relational stability and alliance performance in supply chain. Omega, 36(4), 600-608.

Yu, Y., \& Huo, B. (2018). Supply chain quality integration: relational antecedents and operational consequences. Supply Chain Management, 23(3), 188-206.

Zhong, J., Ma, Y., Tu, Y., \& Li, X.. (2016). Supply chain quality management: an empirical study. International Journal of Contemporary Hospitality Management, 28(11), 2446-2472.

Ziggers, G. W., \& Trienekens, J. (1999). Quality assurance in food and agribusiness supply chains: developing successful partnerships. International Journal of Production Economics, 60-61, 271-279.

Data de submissão: 11 de outubro de 2019

Data de aceite: 23 de novembro de 2020

Classificação JEL: M11 